\title{
$\rho-$ Meson Form-factors in Point form of Poincaré-Invariant Quantum Mechanics
}

Received: 6 December 2020 / Accepted: 13 May 2021 / Published online: 3 June 2021

(C) The Author(s) 2021

\begin{abstract}
Form-factors investigation of $\rho^{ \pm}$-mesons was carried out within the framework of the relativistic quark model, based on point form of Poincaré-invariant quantum mechanics taking into account the internal structure of constituent quarks. It is shown that the parameters of the model obtained from the condition of the agreement of theoretical calculations with experimental data on leptonic decays for light $\pi^{ \pm}$- and $\rho^{ \pm}$-mesons lead to the results correlating with calculations in models based on light-front and instant forms of dynamics. The proposed scheme for the combined description of lepton transitions of pseudoscalar and vector mesons that is based on point form of dynamics, leads to the results on the magnetic moment of $\rho^{ \pm}$-meson correlating with the experimental data and other models.
\end{abstract}

\section{Introduction}

The precision of modern experimental data has renewed interest in studying the mechanism of quarks interaction within hadrons. Light sector mesons, consisting of light $\mathrm{u}$ - and d-quarks are of interest. Among the variety of approaches and models devoted to the description of various characteristics of bound quark-antiquark states, models based on the Poincaré group should be noted. Such models are known to be relativistic [1], which makes their use appropriate for describing the characteristics of light sector mesons.

Currently light-front form is in most common use of three forms [2] of Poincaré-invariant quantum mechanics (further PiQM) to calculate the characteristics of light mesons. This is due to the fact that there are no diagrams of pair production from vacuum in this form of PiQM. The indicated advantage makes it possible to use this form of dynamics successfully both for calculating the lepton characteristics $[3,4]$ and semileptonic and radiative transitions of pseudoscalar and vector mesons $[5,6]$. However, light-front dynamics has a serious disadvantage associated with the rotational invariance of the generators of the dynamic group set [7]. This feature leads to so-called "angular condition" for matrix elements $I_{\lambda^{\prime}, \lambda}\left(\lambda^{\prime}, \lambda\right.$ - helicity of the final and initial particles, respectively) which in particular complicates the calculation of $\rho^{ \pm}$-meson form-factors. So for example matrix element $I_{1,0}$ is used in work [8] to calculate the magnetic moment of $\rho^{ \pm}$-meson. The author of the work [4] related elements $I_{1,0}$ and $I_{0,0}$ for these calculations. Some cases in which the angular condition was not observed at all [7] are also known.

Instant form of dynamics is also used to calculate various characteristics of electroweak decays of pseudoscalar and vector mesons. Magnetic moment calculations $\mu_{\rho}$ in $[9,10]$ lead to the value $\mu_{\rho}=2.10 \pm 0.05$

V. Y. Haurysh $(\varangle)$

Sukhoi State Technical University, Gomel, Republic of Belarus

E-mail:mez0n@inbox.ru

V. V. Andreev

Francisk Skorina Gomel State University, Gomel, Republic of Belarus

E-mail: vik.andreev@rambler.ru 
(in units $e / 2 m_{\rho}$ ). As a special feature of the performed calculations we record the fact of taking into account the anomalous magnetic moments of quarks $\kappa_{u}, \kappa_{d}$.

An obvious advantage of point form of dynamics is the presence of interaction only in the operator of space-time translations, which leads to the Lorentz covariance operator equations and simple behavior of wave functions with respect to Lorentz transformations. However, in point form of PiQM form-factors calculations of pseudoscalar and vector mesons are less common. This is due to the fact that calculations lead to the results that differ from experimental data both within the framework of this form of PiQM and its various modifications. So for example in work [11] the behavior of the $\pi^{ \pm}$-meson form-factor for various variants of masses of constituent quarks was studied: the results differ from the experimental data of work [12]. In [14] $\rho^{ \pm}$-meson form-factor study has been carried out using the quark-antiquark potential, which depending on the value of the masses of constituent quarks, leads to different results on the magnetic moment of the vector particle. It follows from the above, that further development of the indicated form of dynamics is a relevant objective in physics of mesons, without taking into account equations with interaction potentials.

The renewed interest in calculating the form factors of vector particles, as mentioned above, is associated with the renewal of experimental data on light mesons. So, the magnetic moment of $\rho^{ \pm}$-meson was calculated in work [15] based on experimental data from the BaBar collaboration in the energy range from 0.9 to $2.2 \mathrm{GeV}$ from reaction $e^{+} e^{-} \rightarrow \pi^{+} \pi^{-} 2 \pi^{0}$ : the authors have shown that indirect estimates give the value $\mu_{\rho}=2.1 \pm 0.5$. However, this calculation differs from theoretical predictions. It is known that the nonrelativistic $S U(6)$-model, in the absence of interaction, gives the value $\mu_{\rho}=2$ [4]. This equality is also true for VMD-model, where $\rho^{ \pm}$-meson is assumed to be a boson with magnetic moment $\mu_{\rho}=2$. Unlike the listed numerical predictions in light-front calculations in $[4,8,13]$ it was estimated that $\mu_{\rho}<2$, which is also confirmed by the QCD-sum rules [16].

In this work, the authors calculate the electroweak characteristics of mesons in the light sector using point form of PiQM. This form of dynamics is the most natural for describing bound states, since the 4-velocities of particles coincide both with interaction and without it. This advantage does not impose restrictions on the matrix elements $I_{\lambda^{\prime}, \lambda}$ which makes the proposed calculations the most reliable.

In Sect. 2 the authors discuss the basic relations of point form of PiQM. The procedure for obtaining integral representations of the decay constants $P(q \bar{Q}) \rightarrow \ell v_{\ell}, V(q \bar{q}) \rightarrow \ell^{+} \ell^{-}$and $\ell \rightarrow V(q \bar{Q}) v_{\ell}$ is discussed, based on the expressions obtained for the vectors of states of pseudoscalar and vector mesons. On the basis of the obtained expressions, we propose an original scheme for calculating the constituent masses of quarks using the pseudoscalar density constant and current masses of quarks.

In Sect. 3 there is a scheme of obtaining the form-factors of the vector $\rho^{ \pm}$-meson in point form of PiQM for the generalized Breit system. The specified frame made it possible to distinguish the expressions proportional to the electrical and magnetic form factors $G_{C}$ and $G_{M}$ respectively.

In Sects. 4 and 5 we present a calculation of integral representations of form-factors $\rho^{ \pm}$-meson with the subsequent estimation of the magnetic moment of the particle. It is shown that the use of anomalous magnetic moments $u$ - and $d$-quarks obtained from $V(P) \rightarrow P(V) \gamma$ decays leads to the result that correlates with experimental data, as well as with calculations in other forms of dynamics. As a generalization of the proposed scheme, the study of the electric and magnetic form-factor of a vector particle was carried out for various transferred momenta. This study is especially relevant, since currently there are no experimental data on the behavior of the form-factors of vector particles.

As a feature of the performed calculations, we note the agreement with the methodology carried out in point form of dynamics for the processes $V(P) \rightarrow P(V) \gamma$ and $V(P) \rightarrow P(V) \ell^{+} \ell^{-}$taking into account the structural characteristics of constituent $u$ - and $d$-quarks. This circumstance makes the proposed model universal for describing the electromagnetic characteristics of pseudoscalar and vector two-particle quark systems.

\section{Model Description}

The meson state vector with spin $J$, mass $M$ and four-momentum $Q\left(Q^{2}=M^{2}, V=Q / M, V=\left\{V_{0}, \mathbf{V}\right\}\right)$ in point form of PiQM $[17,18]$ in the quark basis $\left|\mathbf{p}_{1}, \lambda_{1}, \mathbf{p}_{2}, \lambda_{2}\right\rangle$ with masses $m_{q}, m_{\bar{Q}}$ is defined by the following expression

$$
|\mathbf{Q}, J \mu, M\rangle=\sum_{\lambda_{1}, \lambda_{2}} \sum_{\nu_{1}, \nu_{2}} \int \mathrm{d} \mathbf{k} \Phi_{\ell S}^{J}\left(\mathrm{k}, \beta_{q \bar{Q}}^{I}\right) \sqrt{\frac{\omega_{m_{q}}\left(\mathrm{p}_{1}\right) \omega_{m_{\bar{Q}}}\left(\mathrm{p}_{2}\right)}{\omega_{m_{q}}(\mathrm{k}) \omega_{m_{\bar{Q}}}(\mathrm{k}) V_{0}}}
$$




$$
\times \Omega\left(\begin{array}{ccc}
\ell \underset{v_{1}}{\ell}, v_{2}, \mu & J
\end{array}\right)\left(\theta_{k}, \phi_{k}\right) D_{\lambda_{1}, v_{1}}^{1 / 2}\left(\mathbf{n}_{W_{1}}\right) D_{\lambda_{2}, v_{2}}^{1 / 2}\left(\mathbf{n}_{W_{2}}\right)\left|\mathbf{p}_{\mathbf{1}}, \lambda_{1}, \mathbf{p}_{2}, \lambda_{2}\right\rangle .
$$

In (1) the notation is introduced

$$
\Omega\left(\begin{array}{ccc}
\ell & S & J \\
v_{1}, & v_{2} & \mu
\end{array}\right)\left(\theta_{k}, \phi_{k}\right)=Y_{\ell m}\left(\theta_{\mathrm{k}}, \phi_{\mathrm{k}}\right) \mathrm{C}\left(\begin{array}{ccc}
s_{1} & s_{2} & S \\
\nu_{1} & \nu_{2} & \lambda
\end{array}\right) \mathrm{C}\left(\begin{array}{ccc}
\ell & S & J \\
m & \lambda & \mu
\end{array}\right),
$$

where $\mathrm{C}\left(\begin{array}{lll}s_{1} & s_{2} & S \\ \nu_{1} & \nu_{2} & \lambda\end{array}\right), \mathrm{C}\left(\begin{array}{ccc}\ell & S & J \\ m & \lambda & \mu\end{array}\right)$ are Clebsch-Gordan coefficients of $S U(2)$ group and $D_{\lambda, v}\left(\mathbf{n}_{W}\right)$ are Wigner's rotation functions [1]. Meson wave function in (1) is subject to the normalization condition

$$
\sum_{\ell, S} \int_{0}^{\infty} \mathrm{dk} \mathrm{k}^{2}\left|\Phi_{\ell S}^{J}\left(\mathrm{k}, \beta_{q \bar{Q}}^{I}\right)\right|^{2}=1
$$

Using expressions

$$
\left\langle 0\left|J^{\mu}\right| \mathbf{Q}, M\right\rangle=i \frac{1}{(2 \pi)^{3 / 2}} \frac{Q^{\mu}}{\sqrt{2 \omega_{M_{P}}(\mathrm{P})}} f_{P},
$$

for pseudoscalar $P(q \bar{Q})$ and

$$
\left\langle 0\left|J^{\mu}\right| \mathbf{Q}, \lambda_{V}, M\right\rangle=i \frac{1}{(2 \pi)^{3 / 2}} \frac{\varepsilon^{\mu}\left(\lambda_{V}\right) M_{V}}{\sqrt{2 \omega_{M_{V}}(\mathrm{P})}} f_{V}
$$

vector $V(q \bar{Q})$ meson respectively in the rest frame of a decaying meson with an electroweak quark current of the form

$$
\left\langle 0\left|\hat{J}_{\mathrm{ew}}^{\mu}\right| \mathbf{k}, \lambda_{1},-\mathbf{k}, \lambda_{2}\right\rangle=\frac{\bar{v}_{\lambda_{2}}\left(-\mathbf{k}, m_{\bar{Q}}\right) \Gamma_{\mathrm{ew}}^{\mu} u_{\lambda_{1}}\left(\mathbf{k}, m_{q}\right)}{(2 \pi)^{3} \sqrt{2 \omega_{m_{q}}(\mathrm{k}) 2 \omega_{m_{\bar{Q}}}(\mathrm{k})}}
$$

after calculating the spinor part and integrating over the solid angle of vector $\mathbf{k}$ it is easy to obtain an integral representation [17] of the decay constants:

$$
\begin{gathered}
f_{I}\left(m_{q}, m_{\bar{Q}}, \beta_{q \bar{Q}}^{I}\right)=\sqrt{\frac{3}{2}} \frac{1}{\pi} \int \mathrm{dk} \mathrm{k}^{2} \Phi\left(\mathrm{k}, \beta_{q \bar{Q}}^{I}\right) \\
\times \sqrt{\frac{W_{m_{q}}^{+}(\mathrm{k}) W_{m_{\bar{Q}}}^{+}(\mathrm{k})}{M_{0}(\mathrm{k}) \omega_{m_{q}}(\mathrm{k}) \omega_{m_{\bar{Q}}}(\mathrm{k})}}\left(1+a_{I} \frac{\mathrm{k}^{2}}{W_{m_{q}}^{+}(\mathrm{k}) W_{m_{\bar{Q}}}^{+}(\mathrm{k})}\right), a_{P}=-1, a_{V}=1 / 3,
\end{gathered}
$$

where

$$
W_{m}^{ \pm}(\mathrm{k})=\omega_{m}(\mathrm{k}) \pm m, \omega_{m}(\mathrm{k})=\sqrt{\mathrm{k}^{2}+m^{2}}, \mathrm{k}=|\mathbf{k}|
$$

and $M_{0}(\mathrm{k})=\omega_{m_{q}}(\mathrm{k})+\omega_{m_{\bar{Q}}}(\mathrm{k})$ represents the invariant mass of two non-interacting quarks [1].

Determination the values of the constituent masses of quarks in our approach, will be conducted using the pseudoscalar density constant $g_{P}$, which is determined from the expression

$$
\left\langle 0\left|J_{5}\right| \mathbf{Q}, M\right\rangle=-i \frac{1}{(2 \pi)^{3 / 2}} \frac{g_{P}}{\sqrt{2 \omega_{M_{P}}(\mathrm{P})}} .
$$

After similar calculations (see [20]) one can obtain an integral representation of the pseudoscalar density constant in point form PiQM:

$$
g_{P}\left(m_{q}, m_{\bar{Q}}, \beta_{q \bar{Q}}^{P}\right)=\sqrt{\frac{3}{2}} \frac{1}{\pi} \int \mathrm{dk} \mathrm{k} \frac{\Phi\left(\mathrm{k}, \beta_{q \bar{Q}}^{P}\right)}{\sqrt{\omega_{m_{q}}(\mathrm{k}) \omega_{m_{\bar{Q}}}(\mathrm{k})}}
$$




$$
\times \sqrt{M_{0}(\mathrm{k})}\left(\sqrt{W_{m_{q}}^{+}(\mathrm{k}) W_{m_{\bar{Q}}}^{+}(\mathrm{k})}+\sqrt{W_{m_{q}}^{-}(\mathrm{k}) W_{m_{\bar{Q}}}^{-}(\mathrm{k})}\right) .
$$

Calculation of constituent masses of quarks and parameters of wave functions will be carried out by solving the system of equations [20]

$$
\left\{\begin{array}{l}
\frac{1}{2}\left(\hat{m}_{u}+\hat{m}_{d}\right)=(3.45 \pm 0.4) \mathrm{MeV} \\
f_{P}\left(m_{u}, m_{d}, \beta_{u \bar{d}}^{P}\right)=f_{P}^{(\text {exp. })} \\
\left(\hat{m}_{u}+\hat{m}_{d}\right) g_{P}\left(m_{u}, m_{d}, \beta_{u \bar{d}}^{P}\right)=f_{P}^{(\text {exp. })} M_{P}^{2}
\end{array}\right.
$$

where $f_{P}^{\text {(exp.) }}, M_{P}$ are experimental values of the decay constant $\pi^{ \pm}$-meson and its mass, $\hat{m}_{q}$ quark current mass [21]. In what follows we will assume that the values of the masses of constituent $u$ - and $d$-quarks are equal $[4,10,11,14,22]$; in this case the solution to the system (9) with oscillator wave function

$$
\Phi\left(\mathrm{k}, \beta_{q \bar{Q}}^{I}\right)=\frac{2}{\pi^{1 / 4}\left(\beta_{q \bar{Q}}^{I}\right)^{3 / 2}} \exp \left[-\frac{\mathrm{k}^{2}}{2\left(\beta_{q \bar{Q}}^{I}\right)^{2}}\right], I=V, P .
$$

leads to the following values of the basic parameters of the model:

$$
m_{u, d}=222.55 \pm 4.90 \mathrm{MeV}, \beta_{u \bar{d}}^{P}=372.49 \pm 11.12 \mathrm{MeV} .
$$

Wave function parameter for vector $\rho^{ \pm}$-meson is determined from the decay of a heavy lepton $\tau^{ \pm} \rightarrow \rho^{ \pm} v_{\tau}$ : using expression (6) and experimental value $f_{\rho^{ \pm}}=210.75 \pm 0.09 \mathrm{MeV}$ [21] one can get

$$
\beta_{u \bar{d}}^{V}=313.10 \pm 0.08 \mathrm{MeV}
$$

Since all the parameters of the model are determined, we proceed to calculating the characteristics of the vector $\rho^{ \pm}$-meson in the proposed model. An original method for calculating the electromagnetic characteristics of vector mesons in point form of dynamics is presented below.

\section{$3 \rho$-meson form-factors in point form of PiQM}

Calculation of the vector meson form-factors of will be conducted from the expression

$$
\begin{gathered}
I_{\lambda^{\prime}, \lambda}^{\mu}=\left\langle\mathbf{Q}^{\prime}, M^{\prime}, \lambda^{\prime}\left|J^{\mu}\right| \mathbf{Q}, M, \lambda\right\rangle=\frac{1}{(2 \pi)^{3}} \frac{1}{\sqrt{V_{0} M_{0}}} \frac{1}{\sqrt{V_{0}^{\prime} M_{0}^{\prime}}}\left(G_{C}\left(q^{2}\right)\right. \\
\times\left(\epsilon^{*}\left(\lambda^{\prime}\right) \cdot \epsilon(\lambda)\right) P^{\mu}+G_{M}\left(q^{2}\right)\left(\left(P \cdot \epsilon^{*}\left(\lambda^{\prime}\right)\right) \epsilon^{\mu}(\lambda)+(P \cdot \epsilon(\lambda)) \epsilon^{* \mu}\left(\lambda^{\prime}\right)\right) \\
\left.G_{\mathcal{Q}}\left(q^{2}\right) \frac{\left(P \cdot \epsilon^{*}\left(\lambda^{\prime}\right)\right)(P \cdot \epsilon(\lambda))}{2 M M^{\prime}} P^{\mu}\right), P^{\mu}=Q^{\mu}+Q^{\prime \mu}, q^{\mu}=Q^{\mu}-Q^{\prime \mu} .
\end{gathered}
$$

In (13) form-factors $G_{C}, G_{M}$ and $G_{\mathcal{Q}}$ at $q^{2} \rightarrow 0$ are defined as charge $e$, magnetic moment $\mu_{\rho}$ and quadrupole moment $\mathcal{Q}_{\rho}$ of $\rho^{ \pm}$-meson [4]:

$$
e_{\rho}=G_{C}(0), \mu_{\rho}=-G_{M}(0), \mathcal{Q}_{\rho}=G_{C}(0)+G_{M}(0)+G_{\mathcal{Q}}(0) .
$$


Table 1 Parametrization for different $\rho^{ \pm}$-meson helicities

\begin{tabular}{llll}
\hline & $\lambda=1$ & $\lambda=0$ & $\lambda=-1$ \\
\hline$\lambda^{\prime}=1$ & $G_{C}\left(q^{2}\right) \times \ell_{1}$ & $G_{M}\left(q^{2}\right) \times \ell_{2}^{(+)}$ & 0 \\
$\lambda^{\prime}=0$ & $G_{M}\left(q^{2}\right) \times \ell_{2}^{(-)}$ & linear combination of $G_{i}\left(q^{2}\right)$ & $G_{M}\left(q^{2}\right) \times \ell_{2}^{(+)}$ \\
$\lambda^{\prime}=-1$ & 0 & $G_{M}\left(q^{2}\right) \times \ell_{2}^{(-)}$ & $G_{C}\left(q^{2}\right) \times \ell_{1}$ \\
\hline
\end{tabular}

For the generalized Breit system $\left(\mathbf{V}_{\mathbf{Q}}+\mathbf{V}_{\mathbf{Q}^{\prime}}^{\prime}=0\right)$ the 4-vector momentums of the initial and final states in point form of dynamics are defined as

$$
\begin{aligned}
Q^{\mu} & =\frac{M}{\sqrt{2}}\left\{\sqrt{1+\varpi_{1,2}}, 0,0, \sqrt{-1+\varpi_{1,2}}\right\} \\
Q^{\prime \mu} & =\frac{M^{\prime}}{\sqrt{2}}\left\{\sqrt{1+\varpi_{1,2}}, 0,0,-\sqrt{-1+\varpi_{1,2}}\right\}
\end{aligned}
$$

where $\left(V_{Q} \cdot V_{Q^{\prime}}^{\prime}\right)=\varpi_{1,2}$. Since 4-vectors polarizations of the initial and final mesons are limited by the conditions $\left(\epsilon^{*} \cdot \epsilon\right)=-1$ and $(Q \cdot \epsilon)=0$ based on expressions (15) one can get:

$$
\begin{aligned}
\epsilon^{\mu}(\lambda) & =\frac{1}{\sqrt{2}}\left(\begin{array}{c}
\left(\lambda^{2}-1\right)^{2} \sqrt{-1+\varpi_{1,2}} \\
\lambda^{2} \\
-\mathrm{i} \lambda \\
\left(\lambda^{2}-1\right)^{2} \sqrt{1+\varpi_{1,2}}
\end{array}\right), \\
\epsilon^{\mu}\left(\lambda^{\prime}\right) & =\frac{1}{\sqrt{2}}\left(\begin{array}{c}
\left(\lambda^{\prime 2}-1\right)^{2} \sqrt{-1+\varpi_{1,2}} \\
\lambda^{\prime 2} \\
-\mathrm{i} \lambda^{\prime} \\
-\left(\lambda^{\prime 2}-1\right)^{2} \sqrt{1+\varpi_{1,2}}
\end{array}\right) .
\end{aligned}
$$

Using expressions (15) - (17) leads the right side of equation (13) to the following (see Table 1) view [23]. In the table we used designations for vectors $\ell_{i}$, defined as

$$
\begin{gathered}
\ell_{1}=\frac{1}{2 M M^{\prime}}\left\{-\left(M+M^{\prime}\right), 0,0,\left(-M+M^{\prime}\right) \sqrt{\frac{\varpi_{1,2}-1}{\varpi_{1,2}+1}}\right\}, \\
\ell_{2}^{( \pm)}=\frac{1}{2} \sqrt{\frac{M^{\prime}\left(\varpi_{1,2}-1\right)}{M}}\{0,1, \pm i, 0\} .
\end{gathered}
$$

The table shows that the form-factors $G_{C}\left(q^{2}\right)$ and $G_{M}\left(q^{2}\right)$ can be calculated from matrix elements $I_{ \pm 1, \pm 1}$ and $I_{1,0} / I_{0,-1}$ respectively.

\section{Form-factor $G_{C}\left(q^{2}\right)$ in Point Form of PiQM}

In the developed model, the quark transition current taking into account (1) and (5) is determined by the following expression [19] :

$$
\begin{aligned}
& I_{\lambda^{\prime}, \lambda}^{\mu}=\frac{1}{4 \pi} \frac{1}{(2 \pi)^{3}} \frac{1}{\sqrt{V_{0} V_{0}^{\prime}}} \sum_{v_{1}, v_{1}^{\prime}} \sum_{\nu_{2}, \nu_{2}^{\prime}} \int \mathrm{d} \mathbf{k} \Phi\left(\mathrm{k}, \beta_{q \bar{Q}}^{V}\right) \mathrm{C}\left(\begin{array}{ccc}
\frac{1}{2} & \frac{1}{2} & 1 \\
\nu_{1} & \nu_{2} & \lambda
\end{array}\right) \mathrm{C}\left(\begin{array}{ccc}
\frac{1}{2} & \frac{1}{2} & 1 \\
v_{1}^{\prime} & v_{2}^{\prime} & \lambda^{\prime}
\end{array}\right) \\
& \times\left(\sqrt{\frac{\omega_{m_{\bar{Q}}}\left(\mathrm{k}_{2}\right)}{\omega_{m_{\bar{Q}}}(\mathrm{k})}} \frac{\bar{u}_{v_{1}^{\prime}}\left(\mathbf{k}_{2}, m_{q}\right) B\left(\boldsymbol{v}_{\mathbf{Q}}\right) \Gamma_{q}^{\mu} u_{v_{1}}\left(\mathbf{k}, m_{q}\right)}{\sqrt{4 \omega_{m_{q}}\left(\mathrm{k}_{2}\right) \omega_{m_{q}}(\mathrm{k})}} D_{\nu_{2}^{\prime}, \nu_{2}}\left(\mathbf{n}_{W_{2}}\right) \Phi\left(\mathrm{k}_{2}, \beta_{q \bar{Q}}^{V}\right)+\right.
\end{aligned}
$$




$$
\begin{array}{r}
+(-1)^{\lambda^{\prime}+\lambda} \sqrt{\frac{\omega_{m_{q}}\left(\mathrm{k}_{1}\right)}{\omega_{m_{q}}(\mathrm{k})}} \frac{\bar{v}_{\nu_{2}}\left(\mathbf{k}, m_{\bar{Q}}\right) B\left(-\boldsymbol{v}_{\mathbf{Q}}\right) \Gamma_{\bar{Q}}^{\mu} v_{v_{2}^{\prime}}\left(\mathbf{k}_{1}, m_{\bar{Q}}\right)}{\sqrt{4 \omega_{m_{\bar{Q}}}(\mathrm{k}) \omega_{m_{\bar{Q}}}\left(\mathrm{k}_{1}\right)}} D_{v_{1}^{\prime}, \nu_{1}}\left(\mathbf{n}_{W_{1}}\right) \\
\left.\times \Phi\left(\mathrm{k}_{1}, \beta_{q \bar{Q}}^{V}\right)\right), \mathbf{k}_{1,2}=\mathbf{k} \pm \boldsymbol{v}_{Q}\left(\left(\varpi_{12}+1\right) \omega_{m_{q, \bar{Q}}}(\mathrm{k})-\mathrm{k} \sqrt{\varpi_{12}^{2}-1} \cos \theta_{k}\right),
\end{array}
$$

where $\boldsymbol{v}_{Q}=\mathbf{V}_{Q} / V_{0}$ and boost operator $B\left(\boldsymbol{v}_{Q}\right)$ is defined by Dirac $\gamma$-matrices [1,2]

$$
B\left(\boldsymbol{v}_{Q}\right)=\frac{I-\left(\boldsymbol{v}_{Q} \cdot \boldsymbol{\gamma}\right) \gamma_{0}}{\sqrt{1-\boldsymbol{v}_{Q}^{2}}} .
$$

Vertex $\Gamma_{q, \bar{Q}}^{\mu}$ in relation (19) in our approach is defined as

$$
\Gamma_{q, \bar{Q}}^{\mu}=F_{1}\left(q^{2}\right) \gamma^{\mu}+F_{2}\left(q^{2}\right) \frac{i \sigma^{\mu \nu}}{2 m_{q, \bar{Q}}} q_{\nu}^{q u a r k}, q^{q u a r k}=k_{1,2}-k
$$

where the form-factors of quarks at $q^{2}=0$ are defined in units $e$ as the total magnetic moment of the quark $\mu_{q, \bar{Q}}$ and its anomalous part:

$$
F_{1}(0)+F_{2}(0)=\mu_{q, \bar{Q}}, \quad F_{2}(0)=\kappa_{q, \bar{Q}}, \quad \mu_{q, \bar{Q}}=e_{q, \bar{Q}}\left(1+\kappa_{q, \bar{Q}}\right) .
$$

The problem of constructing a conserved electromagnetic current resides in the presence of hadronic interactions in point form of PiQM discussed in the work [24]. In our work to fulfill the requirement of preserving the vertex (21) is extended by multiplication by tensor

$$
\Gamma_{q, \bar{Q}}^{\mu} \Rightarrow\left(-g^{\mu \nu}+\frac{q^{\mu} q^{\mu}}{q^{2}}\right) \Gamma_{q, \bar{Q}}^{v}
$$

in this case, the condition for the conservation of the electromagnetic current $\left(q \cdot I_{\lambda^{\prime}, \lambda}\right)=0$ will be executed automatically.

We will carry out form-factor calculation $G_{C}$ out using matrix elements $I_{ \pm 1, \pm 1}$. Calculating the spinor part (19) and integrating over the angles of vector $\mathbf{k}$ at $q^{2} \rightarrow 0$ gives

$$
G_{C}(0)=\int \mathrm{dk} \mathrm{k}^{2}\left|\Phi\left(\mathrm{k}, \beta_{q \bar{Q}}^{V}\right)\right|^{2}\left(e_{q}+e_{\bar{Q}}\right)
$$

(terms proportional $F_{2}\left(q^{2}\right)$ vanish after integration over the solid angle of vector $\mathbf{k}$ ). Since charged $\rho^{ \pm}-$meson consists of $u$ - and $\bar{d}$-quarks from (24), we obtain that, taking into account the normalization of the wave function (see equation (2)) $G_{C}=e_{q}+e_{\bar{Q}}=1$ (in units $e$ ) $[4,11,14,22]$. We will carry out behavior for various $q$ with basic model parameters (see section 2) in comparison with calculations in other forms of dynamics with form-factors [25]

$$
F_{1}\left(q^{2}\right)=\frac{e_{q, \bar{Q}}}{1-\frac{\left\langle\left. r_{q, \bar{Q}}\right|^{2}\right.}{6} q^{2}}, F_{2}\left(q^{2}\right)=\frac{e_{q, \bar{Q}^{\kappa}} \kappa_{q, \bar{Q}}}{\left(1-\frac{\left\langle\left. r_{q, \bar{Q}}\right|^{2}\right.}{12} q^{2}\right)^{2}},
$$

where [26]

$$
\left\langle r_{q, \bar{Q}}\right\rangle^{2}=\frac{a}{m_{q, \bar{Q}}^{2}}, a=0.3 .
$$

Numerical integration with oscillator wave function (10) in the region $q \leq 2 \mathrm{GeV}$ leads to the following form-factor behavior $G_{C}\left(q^{2}\right)$ (the figure also presents calculations in instant form and light-front dynamics):

The analysis of Fig. 1 shows that the calculations in the proposed model, based on point form of PiQM, are in good agreement with the calculations in instant form of dynamics. 


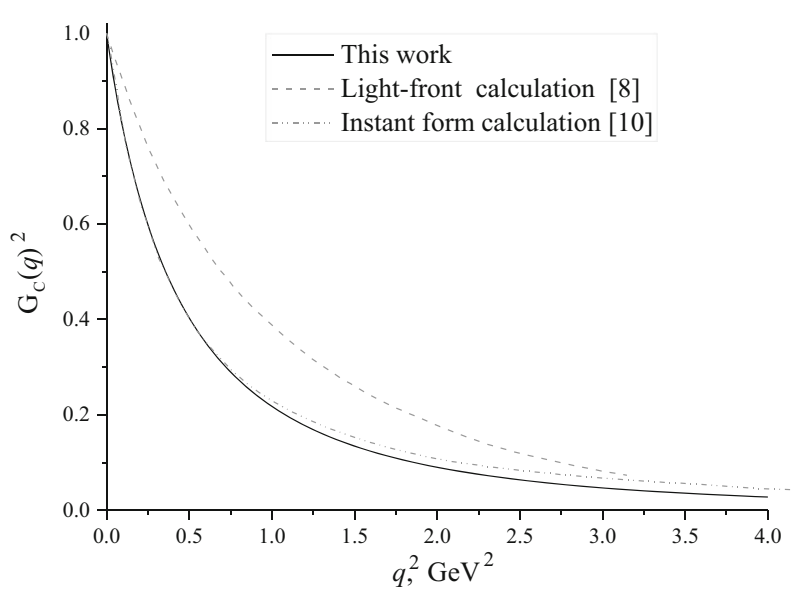

Fig. $1 G_{C}\left(q^{2}\right)$ behaviour in different forms of PiQM

\section{Form-factor $G_{M}\left(q^{2}\right)$ in Point Form of PiQM}

Form-factor $G_{M}$ calculation based on the table 1 can be carried out directly from the matrix elements $I_{1,0} / I_{0,-1}$ (calculations are equivalent). Taking into account the equality of the masses of constituent quarks $m_{q}=m_{\bar{Q}}$ after calculating the spinor part of the expression (19) and integration over the angular variables of vector $\mathbf{k}$, we get an integral representation of the form-factor $G_{M}$ at $q^{2} \rightarrow 0$ taking into account the anomalous magnetic moments of quarks:

$$
\begin{aligned}
& G_{M}(0)=\int \mathrm{dk} \mathrm{k}^{2}\left|\Phi\left(\mathrm{k}, \beta_{q \bar{Q}}^{V}\right)\right|^{2}\left(e_{q} \eta_{1}\left(\mathrm{k}, m_{q}\right)+\frac{e_{q} \kappa_{q}}{2 m_{q}} \eta_{2}\left(\mathrm{k}, m_{q}\right)-\right. \\
& \left.-e_{\bar{Q}} \eta_{1}\left(\mathrm{k}, m_{\bar{Q}}\right)-\frac{e_{\bar{Q}} \bar{Q}_{\bar{Q}}}{2 m_{\bar{Q}}} \eta_{2}\left(\mathrm{k}, m_{\bar{Q}}\right)\right)
\end{aligned}
$$

In equation (27) auxiliary functions are defined by the following expressions:

$$
\begin{gathered}
\eta_{1}(\mathrm{k}, m)=\frac{2}{3}\left(2+\frac{m}{\omega_{m}(\mathrm{k})}\right), \\
\eta_{2}(\mathrm{k}, m)=\frac{4}{3}\left(m+\frac{\omega_{m}^{2}(\mathrm{k})+m^{2}}{\omega_{m}(\mathrm{k})}\right) .
\end{gathered}
$$

Substitution of the values of constituent quark masses and anomalous magnetic moments obtained from decays $V(P) \rightarrow P(V) \gamma($ see [17])

$$
\kappa_{u}=-0.123, \kappa_{d}=-0.088
$$

with wave function (10) leads to the following value of the anomalous magnetic moment $\rho^{ \pm}$-meson in point form of PiQM:

$$
\mu_{\rho}=2.29 \pm 0.01 \text { (in } e / 2 m_{\rho} \text { units). }
$$

The resulting value is comparable to other approaches and models in table 2:

The analysis of the table 2 shows that the obtained value in the proposed model based on point form of $\mathrm{PiQM}$ lies within reasonable limits in comparison with other forms of dynamics, as well as with experimental data of work [15].

We will carry out form-factor $G_{M}\left(q^{2}\right)$ behavior research with different transmitted momentums with $F_{1}\left(q^{2}\right)$ and $F_{2}\left(q^{2}\right)$ (see (25)). Using model parameters from section 2 and anomalous magnetic moments of quarks (30) one can get the following $G_{M}\left(q^{2}\right)$ behavior in the proposed approach:

From Fig. 2 it can be seen that the behavior of the form-factor $G_{M}$ for different $q^{2}$ in the proposed model correlates with instant and light-front form calculations. 
Table 2 Magnetic moment of $\rho^{ \pm}$-meson in different models and approaches

\begin{tabular}{lc}
\hline Model & Value in units $e / 2 m_{\rho}$ \\
\hline Light-front calculation [4] & 1.83 \\
Light-front calculation [7] & 2.10 \\
Instant form calculation [10] & $2.10 \pm 0.05$ \\
Point form calculation [14] & $2.25 \pm 0.05$ \\
Light-front calculation [27] & 2.26 \\
Light-front calculation [28] & 2.20 \\
Experimental data [15] & $2.10 \pm 0.50$ \\
Our & $2.29 \pm 0.01$ \\
\hline
\end{tabular}

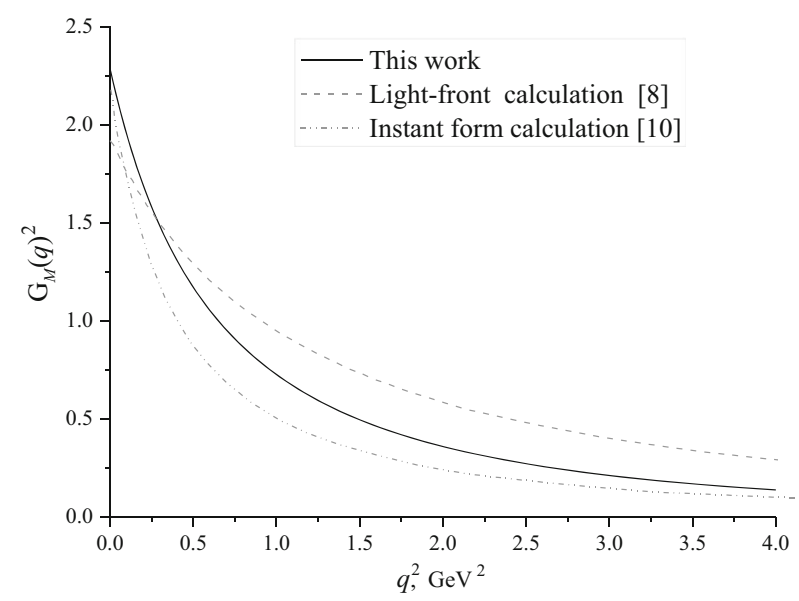

Fig. $2 G_{M}\left(q^{2}\right)$ behaviour in different forms of PiQM

\section{Conclusion and remarks}

The work is dedicated to the problem of electromagnetic $\rho^{ \pm}$-mesons characteristics description in point form of PiQM. In the course of the work, the authors obtained the basic parameters of the model from the leptonic decays of pseudoscalar and vector $\pi^{ \pm}-$and $\rho^{ \pm}$-mesons, which were used to describe the $G_{C}\left(q^{2}\right)$ and $G_{M}\left(q^{2}\right)$ formfactors of vector $\rho^{ \pm}$-mesons. As a feature of the calculations it should be noted that the previously developed technique [17-19] of constructing electromagnetic quark current with spectator quarks led to results on the electromagnetic characteristics of $\rho^{ \pm}$-meson, comparable to experimental and other models. Also in this work, the problem of electromagnetic meson current conservation was solved by introducing the transverse tensor (see Eq. (23)). Despite the cumbersome calculations for the generalized Breit system this method led to compact expressions (see (28), (29)) and physical results comparable to alternative models and experimental data [15].

It also should be noted that the use of the pseudoscalar density constant and the harmonic oscillator wave function for pseudoscalar and vector mesons made it possible not to introduce an explicit form of equations with the potential of the quark-antiquark interaction. The indicated simplicity of the proposed model nevertheless leads to the values of constituent masses of quarks, comparable with other models (see [20]).

The obtained physical results on the electromagnetic characteristics of light mesons make it possible to use the proposed model both for describing hadronic transitions without quark flavour changing $h(q \bar{q}) \rightarrow$ $h_{1}(q \bar{Q}) h_{2}(Q \bar{q})$, radiative decays $h \rightarrow \ell v_{\ell} \gamma$ and for three-particle bound systems studies.

Open Access This article is licensed under a Creative Commons Attribution 4.0 International License, which permits use, sharing, adaptation, distribution and reproduction in any medium or format, as long as you give appropriate credit to the original author(s) and the source, provide a link to the Creative Commons licence, and indicate if changes were made. The images or other third party material in this article are included in the article's Creative Commons licence, unless indicated otherwise in a credit line to the material. If material is not included in the article's Creative Commons licence and your intended use is not permitted 
by statutory regulation or exceeds the permitted use, you will need to obtain permission directly from the copyright holder. To view a copy of this licence, visit http://creativecommons.org/licenses/by/4.0/.

\section{References}

1. B.D. Keister, W.N. Polyzou, Relativistic Hamiltonian dynamics in nuclear and particle physics. Adv. Nucl. Phys. 20, 225-479 (1991)

2. W.N. Polyzou, Y. Huang, Ch. Elster, W. Glockle, J. Golak, R. Skibinski, H. Witala, H. Kamada, Mini review of Poincaré invariant quantum theory. Few Body Syst. 49, 129-147 (2011)

3. Q. Chang, X.N. Li, X.Q. Li, F. Su, Y.D. Yang, Self-consistency and covariance of light-front quark models: testing via $P, V$ and $A$ meson decay constants, and $P \rightarrow P$ weak transition form factors. Phys. Rev. D 98(11) (2018)

4. W. Jaus, Consistent treatment of spin-1 mesons in the light-front quark model. Phys. Rev. D 67 (2003)

5. Q. Chang, X.N. Li, L.T. Wang, Revisiting the form factors of $\mathrm{P} \rightarrow \mathrm{V}$ transition within the light-front quark models. Eur. Phys. J. C. 79(5), $422(2019)$

6. Qin Chang, Yunyun Zhang, Xiaonan Li, Study of $\bar{B}_{u, d, s}^{*} \rightarrow D_{u, d, s}^{*} V\left(V=D_{d, s}^{*-} K^{*-} \rho^{-}\right)$weak decays. Chin. Phys. C 43(10) (2019)

7. B.L.G. Bakker, H.-M. Choi, Chueng-Ryong Ji, Vector meson form-factor analysis in light front dynamics. Phys. Rev. D 65 (2002)

8. H.-M. Choi, Chueng-Ryong Ji, Electromagnetic structure of the $\rho$-meson in the light front quark model. Phys. Rev. D 70 (2004)

9. A.F. Krutov, R.G. Polezhaev, V.E. Troitsky, Electroweak properties of $\rho$-meson in the instant form of relativistic quantum mechanics. EPJ Web Conf. 138, 02007 (2017)

10. A.F. Krutov, R.G. Polezhaev, V.E. Troitsky, Magnetic moment of the $\rho$-meson in instant-form relativistic quantum mechanics. Phys. Rev. D 97 (2018)

11. T.W. Allen, W.H. Klink, Pion charge form factor in point form relativistic dynamics. Phys. Rev. C 58, 3670-3673 (1998)

12. S.R. Amendolia et al., A measurement of the pion charge radius. Phys. Lett. B 146, 116-120 (1984)

13. B.L.G. Bakker, H.-M. Choi, Chueng-Ryong Ji, Transition form-factors between pseudoscalar and vector mesons in light front dynamics. Phys. Rev. D 67 (2003)

14. E.P. Biernat, W. Schweiger, Electromagnetic $\rho$-meson form factors in point-form relativistic quantum mechanics. Phys. Rev. C 89, 055205 (2014)

15. G.D. Garcia, S.G. Toledo, Determination of the magnetic dipole moment of the $\rho$-meson using 4 pion electroproduction data. Int. J. Mod. Phys. Conf. Ser. 35, 1460463 (2014)

16. A. Samsonov, Magnetic moment of the $\rho$-meson in QCD sum rules. Phys. Atom. Nucl. 68, 114-118 (2005)

17. VYu. Haurysh, V.V. Andreev, Electroweak decays of unflavored mesons in Poincaré covariant quark model. Turk. J. Phys. 43(2), 167-177 (2019)

18. VYu. Haurysh, V.V. Andreev, Poincaré-covariant quark model of electroweak light mesons decays. EPJ Web Conf. 204, 08006 (2019)

19. V.V. Andreev, VYu. Haurysh, A.F. Krutov, Method of calculation of electroweak characteristics of mesons in the Poincare invariant quantum mechanics. PFMT. 34, 7-19 (2018)

20. V.V. Andreev, V. Haurysh, Constituent quark masses in Poincaré-invariant quantum mechanics. J. Phys. Conf. Ser. 938(1), 012030 (2017)

21. Particle Data Group and P. A. Zyla, R. M. Barnett, et all, Review of Particle Physics. J.Progress of Theoretical and Experimental Physics. 2020 (8), 2093 (2020)

22. J.P.B.C. Melo, K. Tsushima, In-Medium $\rho$-meson properties in a light-front approach. Few Body Syst. 58(2), 82 (2017)

23. V. Yu. Haurysh, V. V. Andreev, Magnetic moment of $\rho$-meson in point for of Poincare-invariant quantum mechanics. Nonlinear Dynamics and Applications. Proceedings of the Twenty-seven Anniversary seminar NPCS'2020. 26, 100-107 (2020)

24. W.H. Klink, Point-Form electrodynamics and the construction of conserved current operators. Few Body Syst. 33, 99-110 (2003)

25. R. Petronzio, S. Simula, G. Ricco, Possible evidence of extended objects inside the proton. Phys. Rev. D 67, 094004 (2003)

26. F. Cardarelli, I.L. Grach, I.M. Narodetsky, Charge form-factor of $\pi$ - and $K$-mesons. Phys. Rev. D 53, 6682-6685 (1996)

27. F. Cardarelli, I.L. Grach, I.M. Narodetsky, G. Salme, S. Simula, Electromagnetic form-factors of the $\rho$-meson in a light front constituent quark model. Phys. Lett. B 349, 393-399 (1995)

28. J.P.B.C. de Melo, T. Frederico, Covariant and light front approaches to the $\rho$-meson electromagnetic form-factors. Phys. Rev. C 55, 2043 (1997)

Publisher's Note Springer Nature remains neutral with regard to jurisdictional claims in published maps and institutional affiliations. 